\title{
La estandarización como tecnología precautoria globalizada: El caso de las radiaciones no ionizantes
}

María Valeria Berros ${ }^{1}$

\section{Resumen}

La estrategia de estandarización se inscribe como uno de los dispositivos jurídicos que dan cuenta de la emergencia, expansión y desarrollo de la racionalidad precautoria, la cual se halla fundamentalmente ligada al gobierno de riesgos medioambientales y relativos a la salud humana. En este trabajo, planteamos de qué manera subyace, en la determinación de límites de emisión de radiaciones no ionizantes, un proceso de globalización del campo socio-jurídico. Partimos de la idea postulada por el sociólogo Boaventura de Sousa Santos acerca de la existencia / yuxtaposición de diferentes espacios de producción normativa (internacional / nacional / local) con el propósito de establecer cuales son los relacionamientos y articulaciones entre éstos a la luz del caso planteado. Para ello, efectuamos un mapeo de las regulaciones respecto del establecimiento de topes de emisión y exposición enroladas en cada uno de estos espacios, así como también, analizamos cómo operan en los discursos jurisprudenciales que dieron tratamiento a supuestos de hecho vinculados a la temática, específicamente, respecto de la instalación de antenas de telefonía celular. Este análisis se formula como un intento por visibilizar la articulación y ensamblajes, inscriptos en el supuesto concreto de fijación de topes de emisión de radiaciones no ionizantes, a fin de contribuir al debate acerca del proceso de globalización en el campo del derecho.

Palavras-chaves: Globalização. Direito. Direito Ambiental.

\section{Introducción}

En el presente trabajo, proponemos indagar sobre la problemática que se visualiza en la determinación de estándares respecto de la emisión de radiaciones

\footnotetext{
${ }^{1}$ CONICET - UNL, Facultad de Ciencias Jurídicas y Sociales, Centro de Investigaciones en Derecho. Ayudante de Cátedra "Introducción a la Sociología".
} 
no ionizantes, también denominadas campos electromagnéticos ${ }^{2}$. Partimos de este caso, a fin de dar cuenta del proceso de establecimiento de topes de emisión a la luz de su carácter de dispositivo precautorio y la expansión global de la estandarización como estrategia de gobierno de este riesgo.

El supuesto particular seleccionado se analiza como una manifestación del proceso de globalización del derecho. En éste se visibiliza cómo normas jurídicas y sus racionalidades subyacentes se trasladan del ámbito de las agencias de gobierno global hacia los Estados nacionales, ya sea a través de la legislación interna, ya sea a través de decisorios judiciales.

En función de este objetivo, postulamos trabajar a partir de la idea de espacios jurídicos diferenciados "[...] a los que corresponden tres formas de derecho: el derecho local, el derecho nacional y el derecho global [...]"3, a efectos de lograr identificar algunas continuidades e interrelaciones entre los mismos, esto es, entre el espacio jurídico internacional / nacional / local.

Para ello, utilizamos una de las herramientas teóricas que postula Boaventura de Sousa Santos, quien plantea una comparación entre los elementos constitutivos de los mapas y la estructura del derecho. Afirma que

[...] El derecho, esto es, las leyes, las normas, las costumbres, las instituciones jurídicas, es un conjunto de

\footnotetext{
${ }^{2}$ La Organización Mundial de la Salud explica que "[...] Las ondas electromagnéticas son transportadas por partículas llamadas cuantos de luz. Los cuantos de luz de ondas con frecuencias más altas (longitudes de onda más cortas) transportan más energía que los de las ondas de menor frecuencia (longitudes de onda más largas). Algunas ondas electromagnéticas transportan tanta energía por cuanto de luz que son capaces de romper los enlaces entre las moléculas. De las radiaciones que componen el espectro electromagnético, los rayos gamma que emiten los materiales radioactivos, los rayos cósmicos y los rayos $\mathrm{X}$ tienen esta capacidad y se conocen como «radiación ionizante». Las radiaciones compuestas por cuantos de luz sin energía suficiente para romper los enlaces moleculares se conocen como «radiación no ionizante». Las fuentes de campos electromagnéticos generadas por el hombre que constituyen una parte fundamental de las sociedades industriales (la electricidad, las microondas y los campos de radiofrecuencia) están en el extremo del espectro electromagnético correspondiente a longitudes de onda relativamente largas y frecuencias bajas y sus cuantos no son capaces de romper enlaces químicos [...]"ORGANIZACIÓN MUNDIAL DE LA SALUD. ¿Qué son los campos electromagnéticos?. Disponible em: <www.who. int/peh-emf/es/>. Acceso en: 17 jul. 2008.

${ }^{3}$ SANTOS, Boaventura de Sousa. Crítica de la razón indolente: contra el desperdicio de la experiencia. Bilbao: Desclée de Brouwer, 2003. p. 234.
} 
representaciones sociales, un modo específico de imaginar la realidad que, a mi entender, tiene mucha similitud con los mapas. El análisis de tales semejanzas presupone, en un primer momento, que el derecho sea concebido, metafóricamente, como mapa y, en un segundo momento, que la metáfora sea tomada literalmente $[\ldots]^{4}$

En ese sentido, analiza tres características fundamentales de los mapas: la escala, la proyección y la simbolización a los efectos de realizar una "cartografía simbólica del derecho" 5

Es a partir de la idea de escala ${ }^{6}$ que proponemos efectuar un recorrido respecto de la problemática de fijación de estándares de emisión a este tipo de radiaciones, identificando qué elementos representativos de cada una de las escalas encontramos en torno al tema, bajo la idea de que

[...] lo que distingue estas formas de derecho es el tamaño de la escala con que regulan la acción social. El derecho local es una legalidad a gran escala; el derecho nacional - estatal es una legalidad de escala media; y el derecho mundial es una legalidad de pequeña escala $[\ldots]^{7}$

Para efectuar ese recorrido, proponemos dividir el trabajo en tres partes.

En la primera de ellas, hacemos referencia a la estrategia de estandarización como uno de los dispositivos que se inscriben dentro de la lógica precautoria.

En la segunda, analizamos las características más relevantes que presentan los diferentes espacios jurídicos que confluyen en la problemática de la fijación de estándares, enfatizando cómo la construcción global de esta estrategia se manifiesta en nuestro derecho nacional y local. Para ello, indagamos acerca de las normas construidas en los tres niveles; a fin de esclarecer qué tipo de relacionamiento se visualiza entre las diversas escalas.

\footnotetext{
${ }^{4}$ SANTOS, Boaventura de Sousa. Crítica de la razón indolente: contra el desperdicio de la experiencia. Bilbao: Desclée de Brouwer, p. 224.

${ }^{5}$ Ibidem, p. 224.

${ }^{6}$ La escala es "[...] la relación entre la distancia en el mapa y la correspondiente distancia en el terreno”. (MONMONIER, 1981, p. 41) y, como tal, implica una decisión sobre el grado de pormenorización de la representación. Los mapas de gran escala tienen un grado más elevado de pormenorización que los mapas de pequeña escala porque cubren un área inferior, a la que es cubierta, en el mismo espacio de diseño, por los mapas de pequeña escala [...]". SANTOS, op. cit., p. 228.

${ }^{7}$ Ibidem, p. 234.
} 
Por último, y a fines meramente exploratorios, proponemos un breve recorrido en relación a algunos casos de la jurisprudencia en materia de campos electromagnéticos a los fines de detectar en tales discursos cómo operan las diversas escalas jurídicas en el andamiaje argumental que sustenta los decisorios seleccionados.

\section{Acerca de dispositivos precautorios}

\subsection{Esquemas de tratamiento social del riesgo y emergencia de la racionalidad precautoria. Estrategia de estandarización como dispositivo precautorio}

Hacia fines del siglo XX, se problematiza en torno al gobierno de los riesgos, en el contexto de emergencia de la denominada "sociedad del riesgo" ${ }^{8}$ Estos desarrollos se inscriben en circunstancias en que se desatan eventos dañosos de gran magnitud $^{9}$, anteriormente desconocidos, fundamentalmente vinculados con el deterioro medioambiental y relativo a la salud humana. Lo expuesto se desenvuelve en concomitancia con algunas crisis sanitarias, como la crisis de la "vaca loca" en Europa, o el "affaire" de la sangre contaminada ${ }^{10}$ en Francia, que vienen a dar cuenta de algunas consecuencias no previstas del desarrollo científico.

Lo expuesto, genera diversas reflexiones en torno a los dispositivos de gestión de los riesgos existentes, lo cual puede reconstruirse a partir de diversos enfoques. De este modo, se destaca una perspectiva "realista", que asume la idea de riesgo como algo existente ontológicamente, y que se inscribe en el marco de las tecnologías actuariales, la ingeniería, la psicología. Por otra parte, nos encontramos con el enfoque llamado "constructivista", que ha de concebir al riesgo como fenómeno sociocultural, y que "[...] toma como punto de partida la pluralidad y variabilidad en las percep-

\footnotetext{
${ }^{8}$ BECK, Ulrich. La sociedad del riesgo: hacia una nueva modernidad. [S.1]: Paidos. 1998.

${ }^{9}$ En su obra "El derecho a destruir", Martine Rèmond - Gouilloud hace una enumeración de eventos que dan cuenta de "los grandes miedos" a los que nos enfrentamos hacia fines del siglo pasado. Entre estos destaca el caso de "Minamata" (1959); “Torrey Canyon" (1967); “Seveso" (1976); “Amoco Cádiz" (1978); “Three Miles Island” (1979); “Agente Naranja” (1980); “Love Canal” (1980); Bhopal (1984); Chernobyl (1986); Sandoz (1986); "Marea negra de Alaska” (1989). RÈMOND-GOUILLOUD, Martine. El derecho a destruir: ensayo sobre el derecho del medio ambiente. Buenos Aires: Lozada, 1994.

10 Consultar HERMITT, Marie-Angèle. La sang et le droit. Paris: Seuil, 1998.
} 
ciones y evaluación de riesgo que sostienen los agentes sociales [...]"11 Dentro de la lógica constructivista, se puede hacer una diferenciación según se trate de un constructivismo "débil", en el que se enrolaría a Ulrich Beck; o de un constructivismo “fuerte", en el que se ubicaría a François Ewald, Pat O’ Malley, o la mirada que ofrecen los estudios sobre ciencia, tecnología y sociedad (CTS).

Cada una de estas perspectivas da cuenta de un singular enfoque respecto de hipótesis de hecho posiblemente dañosas que se inscriben a modo de consecuencia no prevista, no deseada o no determinable de intervenciones humanas. Esta característica de indeterminación se torna trascendente dado que genera la necesidad de repensar la lógica de funcionamiento y relacionamiento de los dos pilares constitutivos de la modernidad: la ciencia y el derecho, las dos "estatuas modernas"12, edificadas a partir de la idea - ¿utópica o real? - de certeza.

Alejada de la noción de certidumbre, la ciencia ya no se presenta como discurso experto homogéneo que puede dar acabadas explicaciones respecto de diversas problemáticas, tal y como se edificó modernamente, sino que comienza a transitar un escenario incierto en que las explicaciones unánimes dan paso a situaciones de controversia y desacuerdo. Se inicia una deconstrucción de la noción de ciencia como " $[. .$.$] dominio de las certezas, de las constantes verificables a partir de la expe-$ riencia $[\ldots]$ " ${ }^{13}$. Se sostiene, por el contrario, que "[...] la ciencia moderna ha cambiado su fisonomía: de una ciencia que puede ser calificada de soberbia, pretenciosa e infalible, lentamente se ha pasado a una ciencia que se reconoce a sí misma como falible y más prudente $[\ldots]]^{14}$

En razón de ello, el esquema de "unicidad del discurso experto" da paso a un "conglomerado de discursos expertos", que podrían graficarse a partir de la idea de "parlamentariedad de la ciencia" ${ }^{15}$ Se inicia un camino en que se propone recrear la

${ }^{11}$ CARR, S; IBARRA, A. Las construcciones del riesgo en Gobernar los riesgos: ciencia y valores en la sociedad del riesgo. Madrid: Biblioteca Nueva OEA, 2004.

${ }^{12}$ SANTOS, Boaventura de Souza. Crítica de la razón indolente: contra el desperdicio de la experiencia. Bilbao: Desclée de Brouwer, 2003.

${ }^{13}$ PRIEUR, 2001, p. 10.

${ }^{14}$ SOZZO, Gonzalo (Coord.). Repensar la regla de la asunción del riesgo: de causal de justificación a dispositivo tecnológico de gobierno de los riesgos. Revista de Derecho de Daños, Santa Fé, v. 1. n. 2, p. 287-343, 2007.

${ }^{15}$ LATOUR, Bruno. Nous n'avons jamais été moderne: essai d’anthropologie symétrique. Paris: La Decouverte, 1997. 
consubstanciación entre "ciencias" y "derecho", lo cual se desprende de la naturaleza misma de la idea de precaución, que propone el establecimiento de un nuevo diálogo. El derecho, ya no logra funcionar adecuadamente a modo de racionalizador de segundo orden. ${ }^{16}$ Por el contrario, se inicia una ruptura de esa relación refleja y se ponen en evidencia las limitaciones que reviste la estructura jurídica moderna al momento de lidiar con la incerteza o la controversia científica. Ello abre la puerta a la creatividad, a la necesidad de "re-pensar", "re-crear", o bien "des-pensar" e "inventar" dispositivos jurídicos cuya naturaleza permita dar cuenta de estas situaciones.

En este marco, la racionalidad precautoria se ha constituido como un nuevo cimiento para el diseño de estos dispositivos y, a su vez, se encuadra como un nuevo fundamento de la responsabilidad ${ }^{17}$.

Dentro de esta lógica, en la obra de F. Ewald ${ }^{18}$, se presenta la idea de precaución como sustento de un nuevo paradigma ${ }^{19}$ de tratamiento social del riesgo, al

${ }^{16}$ SANTOS, Boaventura de Souza. Crítica de la razón indolente: contra el desperdicio de la experiencia. Bilbao: Desclée de Brouwer, 2003.

${ }^{17}$ Los basamentos filosóficos de la responsabilidad han sido analizados por Hans Jonas en su obra "Le principe de responsabilité". En ella, el autor divide la historia de la humanidad a partir de la ruptura entre mundo antiguo y mundo moderno, considerando que en la relación con la naturaleza se establece un pasaje del hombre como ser dentro de la naturaleza al hombre como dueño y señor de la misma, a partir del desarrollo de la ciencia y tecnología. De esta manera, el individuo adquiere una capacidad de destrucción, tanto de sí mismo como de su entorno, anteriormente desconocida, lo que genera necesariamente el nacimiento de un nuevo concepto de responsabilidad acorde a ese poder ilimitado, que, en este caso, estaría dado por la idea de precaución. JONAS, Hans. Le principe de responsabilité. Paris: Du Cerf, 1990.

${ }^{18}$ Consultar EWALD, François. L’Etat Providence. Paris: Grasset, 1986; “Responsabilité, solidarité, sécurité” publicado en Risques. Assurance, droit, responsabilité. № 10 Paris. Avril - Juin 1992 ; "Philosophie de la précaution" publicado en L’Année sociologique. N 46 - 2. Paris. 1996 ; "Le retour du malin génie. Esquisse d'une philosophie de la précaution" publicado en "Le principe de précaution dans la conduite des affaires humains" sous la direction Olivier Godard. Editorial de la Maison des Sciences de l'homme. Paris. 1997 ; "Le principe de précaution". Que sais je ? PUF. Paris. 2001 ; "La société du risque" en "Une nouvelle modernité ? Traitements de surface et exploration des profondeurs". Cahier LaSer N ${ }^{\circ}$ 7. Descartes \& Cie. Paris. 2001; “L' État de précaution ” en Rapport public du Conseil de Etat. Jurisprudence et avis de 2004. Responsabilité et socialisation du risque. La Documentation Française. Etudes et documents $N^{\circ}$ 56. 2005.

${ }^{19}$ Remarcamos que el uso "paradigmas" se justifica por su utilidad a los efectos de construir categorías analíticas. Cada uno de los paradigmas no reemplaza definitivamente al anterior sino que en cada uno de ellos se presentan sedimentaciones importantes de los paradigmas anteriores. 
que denomina "paradigma de la seguridad", y que se distingue de dos paradigmas precedentes, el de "responsabilidad"20 y el de "solidaridad"21.

Este paradigma de "seguridad", surgido hacia fines del siglo XX, se asienta en la aparición de casos inmersos en un contexto de incerteza o controversia científica, en el que se destaca la posibilidad eventual de daños graves o irreversibles. Éstos encauzan un retorno a las catástrofes, ya no provenientes de la naturaleza, sino de la misma actividad del hombre ${ }^{22}$ o, lo que en palabras de Ulrich Beck, se denomina proliferación de "riesgos manufacturados" 23

Uno de los dispositivos que se inscriben en esta lógica es el principio de precaución, que se construye como relevante dentro del marco del derecho ambiental, que lo presenta como uno de sus principios fundamentales. Este postula la adopción de medidas efectivas ante el posible acaecimiento de daños graves o irreversibles ${ }^{24}$, más allá de la certeza de su producción. El principio ha sido postivi-

${ }^{20}$ F. Ewald construye el paradigma de "responsabilidad" a partir de la idea de culpa como factor atributivo de la responsabilidad, concibiendo a ésta como un regulador primordial de las conductas humanas. EWALD, François. Le retour du malin génie: esquisse d'une philosophie de la précaution. In: GODARD, Olivier (Ed.). Le principe de précaution dans la conduite des affaires humains. Paris: Maison des Sciences de l'homme, 1997. p. 99-126.

${ }^{21}$ F. Ewald presenta el paradigma de "solidaridad" a partir de la idea de desarrollo y expansión de la técnica del seguro como mecanismo de repartición de riesgos. Ibidem.

${ }^{22}$ EWALD, F. Le retour du malin génie: esquisse d'une philosophie de la précaution. In: GODARD, Olivier (Ed.). Le principe de précaution dans la conduite des affaires humains. Paris: Maison des Sciences de l'homme, 1997.

${ }^{23}$ BECK, Ulrich. La sociedad del riesgo mundial. [S. 1.]: Paidós, 2007.

${ }^{24}$ Respecto de las nociones de gravedad o irreversibilidad cabe señalar que en los diferentes textos que han acogido el principio precautorio, hay disímiles formulaciones. Algunas de ellas requieren de la existencia de gravedad o irreversibilidad indistintamente, como la Declaración de Río sobre Medio Ambiente y Desarrollo en su principio número 15, así como también en la Ley 25.675/2002 de Política Ambiental Argentina. En cambio, por otro lado, existen definiciones en las que se requiere de ambos aspectos, como sucede en el caso de la ley francesa 95 - 101 "Renforcement de la Charte de l'Environnement". Por otra parte, destacamos la propuesta de definición que efectuó la UNESCO, en febrero de 2005, en su «Informe del Grupo de Expertos sobre el Principio de Precaución » (entre cuyos integrantes se encuentra la Dra. Aida Kemelmajer de Carlucci) y en el que se propuso una definición que incorpora un criterio ético: "Cuando las actividades humanas corren e riesgo de conducir a un daño moralmente inaceptable que es científicamente plausible pero incierto, diversas medidas pueden ser tomadas para evitar o disminuir la posibilidad de ese daño". Este componente moral también es puesto de relieve en las « Guías de actuación para el principio precautorio " propuestas por la UICN (Unión Internacional para la Conservación de la Naturaleza). 
zado, en un primer momento, en normativas internacionales ${ }^{25}$ y regionales ${ }^{26}$, para luego ser plasmado en normas nacionales ${ }^{27}$.

Dentro del marco de las tecnologías precautorias, otra de sus manifestaciones trascendentes se advierte en el procedimiento de determinación de estándares.

\footnotetext{
${ }^{25}$ Entre los instrumentos de alcance universal se puede establecer una distinción según su carácter vinculante o no vinculante. Dentro de los primeros, hallamos la Convención sobre Conservación de las Especies Migratorias de Animales Silvestres (1979), la Convención de Naciones Unidas sobre el Derecho del Mar (1982), el Convenio de Viena para la Protección de la Capa de Ozono (1985), el Protocolo de Montreal Relativo a Sustancias que agotan la Capa de Ozono (1987), el Convenio sobre el Control de los Movimientos Transfronterizos de los Desechos Peligrosos y su Eliminación (1989), la Convención Marco de las Naciones Unidas sobre Cambio Climático (1992), el Convenio sobre Diversidad Biológica (1992), el Protocolo de Cartagena sobre Seguridad de la Biotecnología (2000), el Acuerdo de la OMC sobre la Aplicación de Medidas Sanitarias y Fitosanitarias (1993), el Acuerdo para la Implementación de las Disposiciones de la Convención de Naciones Unidas sobre Derecho del Mar de 10 de diciembre de 1982 Relativo a la Conservación y Manejo de las Especies Transzonales y Altamente Migratorias (1995), la Convención conjunta sobre Seguridad en la Gestión del Combustible Gastado y sobre Seguridad en la Gestión de Desechos Radiactivos (1997) y el Convenio sobre Contaminantes Orgánicos Persistentes (2001). Dentro de los instrumentos no vinculantes de alcance universal, hallamos la Declaración de Estocolmo sobre Medio Humano (1972), la Carta Mundial de la Naturaleza (1982), el Informe del Consejo de Gobierno del Programa de Naciones Unidas para el Medio Ambiente en su decimoquinto período de sesiones (1989), la Declaración de Río sobre Medio Ambiente y Desarrollo (1992), la denominada "Agenda 21" (1992), el Código de Conducta de Pesca Responsable de la FAO (1995). DRNAS, Zlata de Clement. El principio de precaución ambiental: la práctica argentina. Córdoba: Lerner SRL, 2007.

${ }^{26}$ Entre los instrumentos de alcance regional se destacan, como vinculantes, la Convención de Bamako sobre la Prohibición de la Importación a África de Desechos Peligrosos (1991); el Convenio sobre la Protección y Utilización de los Cursos de Agua Transfronterizos y de los Lagos Internacionales (1992); la Convención sobre la Protección del Medio Marino en el Mar Báltico (1992); la Convención de Oslo y París para la Protección del Medio Marino en el Noreste Atlántico (1992); el Tratado de la Unión Europea (Maastricht 1992, Ámsterdam 1997, Niza 2001); la Convención sobre la Conservación y Administración de los Recursos en el Mar de Bering Central (1994); la Convención de Barcelona para la Protección de Medio Marino y de las Regiones Costeras del Mediterráneo (1995); el Acuerdo Marco para la Conservación de los Recursos Vivos Marinos en el Alta Mar del Pacífico Sur-Acuerdo de Galápagos (2000); la Convención sobre la Conservación y Manejo de las Especies Altamente Migratorias en el Océano Pacífico Oeste y Central (2000); la Convención sobre la Conservación y Manejo de los Recursos Pesqueros en el Atlántico Sur (2001), el Acuerdo Marco sobre Medio Ambiente del MERCOSUR (2001). Asimismo, como instrumentos regionales no vinculantes hallamos la Declaración Ministerial de la Primera Conferencia sobre Protección del Mar del Norte (1984), la Declaración de la Segunda Conferencia sobre la Protección del Mar del Norte (1987) y la Declaración de la Tercera Conferencia sobre la Protección del Mar del Norte (1990). (Drnas, 2007).

${ }^{27}$ En el caso de Argentina, el mismo se positiviza en el año 2002 con la sanción de la Ley de Política Ambiental Nacional Na 25.675.
} 
Ello se fundamenta en que la ausencia de certezas no puede convertirse en pretexto de demora para la implementación de medidas de protección respecto de hipótesis de hecho posiblemente dañosas. Por el contrario, las autoridades públicas se ven en la necesidad de tomar decisiones antes de contar con informaciones completas, razón por la cual las decisiones a adoptar son intrínsicamente revisables ${ }^{28}$.

En el caso que nos ocupa, la estrategia de implementación de estándares de emisión se puede verificar en los tres niveles a partir de los cuales trabajamos, tal y como se señala en el apartado siguiente, lo cual permite dar cuenta de un proceso de expansión desde agencias internacionales respecto del derecho estatal y local.

\subsection{Recomendaciones globales para habilitaciones locales}

En los tres apartados que se desarrollan a continuación, intentaremos visibilizar algunos de los ensamblajes que se presentan entre los tres espacios jurídicos bajo estudio. Lo hacemos a partir de la idea de "constelación jurídica" ${ }^{29}$ en la que se presentan articulaciones entre los diferentes espacios regulatorios en un determinado tiempo y lugar. Desde esta perspectiva, proponemos recorrer las regulaciones referidas a los estándares de emisión de los espacios internacional / nacional / local.

\section{Sobre la expansión de la "pequeña escala".}

A efectos de rastrear, en el marco del espacio jurídico internacional, la regulación respecto de la problemática de los campos electromagnéticos, se hace necesario señalar algunas normativas, presentadas a modo de recomendaciones, encuadradas en el derecho "soft".

Estas se inscriben en un nuevo espacio normativo de carácter no esta$\operatorname{tal}^{30}$. De este modo, desde el año 1977, dentro de la Asociación Internacional

\footnotetext{
${ }^{28}$ DE SADELEER, Nicolás. Environnemental principles: from political slogans to legal rules. New York: Oxfor University Press, 2002.

${ }^{29}$ Esta idea fue trabajada por el Dr. Gonzalo Sozzo en su Seminario "Globalización y Derecho" desarrollado en el marco del CAI+D (FCJS - UNL) del mismo nombre, los días 11 y 12 de agosto en la Facultad de Ciencias Jurídicas y Sociales de la Universidad Nacional del Litoral.

${ }^{30}$ SANTOS, Boaventura de Sousa. A globalizacao e as ciencias sociais. Sao Paulo: Cortéz , 2002.
} 
para la Protección contra la Radiación (en adelante IRPA), se desarrolla un grupo de trabajo que efectúa estudios acerca de los posibles efectos dañosos provenientes de radiaciones no ionizantes.

Este grupo se denomina Comisión Internacional para las Radiaciones No Ionizantes (en adelante ICNIRP) y trabaja en cooperación con la División de Salud Ambiental de la Organización Mundial de la Salud (en adelante OMS), específicamente, en el Programa de Criterios de Salud Ambiental de la OMS, auspiciado por el Programa de Naciones Unidas para el Ambiente (en adelante UNEP).

La ICNIRP es un cuerpo formado por expertos independientes de disciplinas diversas ${ }^{31}$ que se eligen por parte de la Comisión de la ICNIRP - a partir de las reglas dispuestas en su carta constitutiva - de entre los expertos de los diferentes cuerpos nacionales de científicos en el área ${ }^{32}$, presentándose como un organismo independiente ${ }^{33}$. Su principal finalidad es la de difundir

${ }^{31} \mathrm{El}$ sitio web de ICNIRP (www.icnirp.de) informa que los expertos que integran este organismo son médicos, dermatólogos, epidemiólogos, biólogos, fisiólogos, físicos e ingenieros; lo cual nos permitiría esbozar la idea de que la lógica a partir de la cual se piensa el problema de los campos electromagnéticos se construye a partir de los parámetros provenientes de las denominadas "ciencias duras". Acceso en fecha: 01 set. 2008.

${ }^{32}$ En este momento los catorce miembros de la ICNIRP pertenecen a los siguientes países: Italia (Paolo Vecchia), Finlandia (Maila Hietanen), Suiza (Anders Alhbom y Per Soderbërg), Alemania (Eckhard Breitbart y Rüdiger Matthes), Holanda (Frank de Gruijl), Estados Unidos (James Lin y Bruce Stuck), Filipinas (Agnette Peralta), Reino Unido (Richard Sawnders y Anthony Swerdlow), Japón (Masao Taki) y Francia (Bernard Veyret).

${ }^{33}$ En el sitio web de la ICNIRP (www.icnirp.de) en un parágrafo se aclara cual es la fuente de obtención de fondos de la siguiente forma: "[...] Where does ICNIRP get its funds ICNIRP is a nonprofit making organisation and is legally registered as such in Germany. All its modest income is used to offset the year-on-year costs of its various activities including carrying out its scientific programme, organising scientific meetings and producing scientific publications. Its income derives from various sources with the exception of industry. In the same way that individual ICNIRP Commission members cannot be employed by industry, ICNIRP as an organisation does not accept funding from industry. The regular income that ICNIRP receives is an annual grant from IRPA. It also receives support from national governments, most notably from the German Environment Ministry for ICNIRP's Scientific Secretariat based in Munich. All other income is generated by the Commission through contract work (to the exclusion of any work for industry), organisation of scientific meetings and sales of its scientific publications. Currently, ICNIRP's contract income comes from contracts placed by various organisations including, the European Commission, EC, the WHO (contract to carry out scientific reviews of the epidemiology, biology and physics and engineering aspects of exposure to extremely low frequency electric and magnetic fields). ICNIRP also receives income from the sales of its publications that defray some of the cost of producing these. ICNIRP members are not paid for their work for the Commission - it 
información así como, también, advertir acerca de posibles daños causados por exposición a radiaciones no ionizantes.

En ese marco, ha efectuado una serie de documentos en los que se evalúan los posibles riesgos relativos a la salud humana provenientes de la exposición a radiaciones no ionizantes, a través de un análisis de los estudios en relación a los efectos biológicos, así como también, respecto de sus características de funcionamiento y criterios de medición de campos electromagnéticos.

A partir de esta información, la ICNIRP presentó una serie de límites de emisión en base a los cuales ha establecido recomendaciones internacionales.

Las primeras recomendaciones se publicaron hacia 1988 y 1990, pero fueron reemplazadas recientemente. Actualmente se sostiene que las

[...] recomendaciones para limitar la exposición, han sido desarrolladas siguiendo una revisión exhaustiva de toda la literatura científica publicada. Los criterios aplicados en el curso de las revisiones fueron diseñados para evaluar la credibilidad de los diversos hallazgos reportados ${ }^{34}$; sólo efectos establecidos, fueron usados como la base para restricciones de la exposición propuestas [... ${ }^{35}$

En esta lógica, la OMS publica el "Marco para el desarrollo de estándares de CEM (campos electromagnéticos) basados en la salud"36 a manera de "manual de instrucciones" para que los diferentes Estados miembros adopten estándares similares de emisión y exposición a radiaciones no ionizantes, lo cual fundamenta en que

[...] Las grandes diferencias entre los límites nacionales y las recomendaciones internacionales pueden fomentar confusión entre los reguladores y responsables de la formulación de políticas, incrementar la ansiedad del público y plantear un desafío a los fabricantes y operadores de sistemas de comunicaciones que necesitan adaptar sus

is entirely voluntary. Only travel and necessary costs for attendance at meetings are reimbursed to members [...]" Acceso en fecha: 02 set. 2008.

${ }^{34}$ Repacholi y Stolwijk 1991; Repacholi y Cardis 1997.

${ }^{35}$ COMITÉ INTERNACIONAL PARA LAS RADIACIONES NO-IONIZANTES. Recomendaciones para limitar la exposición a campos eléctricos, magnéticos y electromagnéticos (hasta $300 \mathrm{GHz}$ ). Disponible em: <www.icnircp.de>. Acceso en: 21 jul. 2008.

${ }^{36}$ ORGANIZACIÓN MUNDIAL DE LA SALUD. [Home page]. Disponível em: <www.who. int> Acceso em: 19 jul. 2008. 
productos a cada mercado. Estos factores han motivado a la Organización Mundial de la Salud (OMS) a establecer un Marco para desarrollar estándares de exposición de CEM basados en la salud empleando un proceso racional conducido científicamente $[\ldots]^{37}$

En este sentido, a los fines de determinar cuales son propiamente los estándares recomendados,

[...] La OMS respalda las recomendaciones de la Comisión Internacional de Protección Contra las Radiaciones No Ionizantes (ICNIRP) y alienta a los Estados Miembros a adoptar estas recomendaciones internacionales. Sin embargo, si un Estado Miembro desea desarrollar sus propios estándares, este Marco puede ser usado como guía [...]. El Marco para el Desarrollo de Estándares de CEM Basados en la Salud asesora sobre como desarrollar límites de exposición basados en la ciencia que protejan la salud de la población de la exposición a los CEM. Este Marco está dirigido a los organismos reguladores y/o de asesorías nacionales que están desarrollando estándares nuevos para los CEM o revisando las bases de sus estándares existentes $[\ldots]^{38}$

Dentro de este marco, entonces, podemos visualizar cómo las investigaciones de la ICNIRP se constituyen en base para el establecimiento de estándares que son recomendados por parte de la OMS a sus Estados miembros.

A su vez, plantea que “[...] Los Estados Miembros que no desean adoptar estándares internacionales deberían considerar cuidadosamente las razones para ello y el valor de desarrollar sus propios estándares antes de embarcarse en este largo proceso [...]"39 y agrega a lo antedicho una serie de preguntas que deberían plantearse los Estados al momento de fijar estándares nacionales diferentes de los recomendados internacionalmente. Es así como plantea las siguientes indagaciones:

[...] ¿Los estándares internacionales realmente no proporcionan una protección adecuada?; ¿Cuál es el beneficio agregado para la salud al desarrollar estándares

\footnotetext{
${ }^{37}$ ORGANIZACIÓN MUNDIAL DE LA SALUD. Marco para el desarrollo de estándares de CEM basados en la salud: framework for developing health-based EMF standards. Trad. Víctor Cruz Ornetta. Lima: INICTEL-UNI, 2007.

${ }^{38}$ Ibidem.

${ }^{39}$ Ibidem.
} 
nacionales?; ¿Es el desarrollo de un estándar nacional diferente, más exigente y de los procedimientos de cumplimiento adicionales verdaderamente costo efectivo tanto desde la perspectiva de la salud pública y de la implementación?; ¿Serán los límites más conservadores, una barrera para la introducción de nuevas tecnologías, las cuales podrían tener beneficios significativos para la salud y para el comercio internacional?; Si el motivo esencial proviene de la preocupación del público ¿La existencia e implementación de estas nuevas regulaciones o directrices aliviarán el problema? [... $]^{40}$

Por otra parte, y dentro de una lógica similar, la OMS presenta el documento "Legislación modelo para la protección contra campos electromagnéticos" basado en que

[...] La necesidad de desarrollar una Legislación Modelo que permita a las agencias gubernamentales limitar la exposición de las personas a los Campos Electromagnéticos (CEM) fue expresada por los miembros del Comité Consultivo Internacional (IAC) al Proyecto Internacional CEM de la Organización Mundial de la Salud (OMS). Dicha legislación facilitaría la introducción de medidas apropiadas para proteger al público y trabajadores de los efectos adversos potenciales de los CEM. Para asistir a los países que no tengan una legislación apropiada para proteger a su población, el Proyecto Internacional CEM ha desarrollado un Acta Modelo y una Regulación Modelo que proporciona el marco legal para brindar dicha protección [...]. Comprende tres elementos: Un Acta Modelo que permita a una Autoridad iniciar regulaciones y estatutos que limiten la exposición de su población a los CEM en el rango de frecuencias de $0 \mathrm{~Hz}$ a $300 \mathrm{GHz}$. Una Regulación Modelo que establezca en detalle el alcance, aplicación, límites de exposición y procedimientos de cumplimiento que son permitidos por el Acta para limitar la exposición de las personas a los CEM. Un Memorando aclaratorio explicatorio describiendo el enfoque del Acta y sus Regulaciones. Si una autoridad nacional desea desarrollar sus propios límites de exposición, debería usar o tomar en cuenta el Marco de Desarrollo de los Estándares de CEM de la OMS [... $]^{42}$

${ }^{40}$ ORGANIZACIÓN MUNDIAL DE LA SALUD. Marco para el desarrollo de estándares de CEM basados en la salud: framework for developing health-based EMF standards. Trad. Víctor Cruz Ornetta. Lima: INICTEL-UNI, 2007.

${ }^{41}$ Idem. [Home page]. Disponível em: <www.who.int> Acesso em: 19 jul. 2008.

${ }^{42}$ Fragmento de la publicación Idem. Legislación modelo para la protección contra campos electromagnéticos: model legislation for electromagnetic fields protection”. Trad. Víctor 
Este documento se encuentra consubstanciado con el anterior, debido a que expresamente remite a los estándares internacionales que establece la ICNIRP, lo que permite hacer referencia al problema de la juridización del discurso experto.

En el caso del Acta Modelo, se afirma que

[...] Para los propósitos de esta Acta, las recomendaciones de la Comisión Internacional de Protección contra las Radiaciones No Ionizantes (ICNIRP) con respecto a las Restricciones Básicas y Niveles de Referencia serán adoptadas como los Límites de Exposición a los CEM relevantes[...] El Ministro tendrá la potestad de introducir regulaciones implementando las recomendaciones específicas de ICNIRP, las medidas necesarias de cumplimiento así como cualquier otro requerimiento que dará un efecto adicional a las disposiciones pertinentes de esta Acta $[\ldots]^{43}$.

A su vez, en el anexo del Acta Modelo, se prevé la incorporación de las “[...] tablas relevantes de las Recomendaciones ICNIRP, versión 1998 o la más reciente [...]”44

De este modo, podemos visualizar como en la "pequeña escala" se construye una propuesta de estandarización con aspiraciones globales, a partir de una lógica propositiva a fin de introducir sus parámetros en la legislación interna de los Estados miembros. Ello se acompaña de toda una serie de elementos dentro del discurso de esta agencia global que remiten a la necesidad de “justificar” la no aplicación de los parámetros que presenta como adecuados, tal y como se expresa en los parágrafos precedentes.

\subsection{Sobre la "escala media". Acerca de las resoluciones nacionales}

En el caso del derecho nacional, encontramos varias normativas en las que se enraíza la "estrategia de estandarización" y en las que se manifiestan los cánones a partir de los que se edifican las recomendaciones establecidas por la ICNIRP.

En primer término, referimos al "Estándart Nacional de Seguridad para la exposición a radiofrecuencias comprendidas entre $100 \mathrm{KHz}$ y $300 \mathrm{GHz}$ ", aprobado

Cruz Ornetta et al. Lima: INICTEL-UNI, 2007. Data de acesso: 19 jul. .2008.

${ }^{43}$ Fragmento de la publicación Idem. Legislación modelo para la protección contra campos electromagnéticos: model legislation for electromagnetic fields protection”. Trad. Víctor Cruz Ornetta et al. Lima: INICTEL-UNI, 2007. Data de acesso: 19 jul. 2008.

${ }^{44}$ Ibidem. 
por Resolución del Ministerio de Salud y Acción Social N a 202/1995, de conformidad con lo establecido en el "Manual de Estándares de Seguridad para la exposición a radiofrecuencias comprendidas entre $100 \mathrm{KHz}$ y $300 \mathrm{GHz}$ y "Radiación de radiofrecuencias: consideraciones biofísicas, biomédicas, y criterios para el establecimiento de estándares de exposición”, Volúmenes I y II, respectivamente, de prospección de radiación electromagnética ambiental no ionizante.

Aquí se establecen los límites máximos de irradiación, lo que deviene de cumplimiento obligatorio según la Resolución N 530/2000 de la Secretaría de Estado de Comunicaciones. Asimismo, la Resolución N 3690/2004 de la Comisión Nacional de Telecomunicaciones, establece en su artículo 4 que

[...] Los sistemas irradiantes que no cumplan con los valores máximos establecidos en la Resolución $N^{\circ}$ 202/1995 del Ministerio de Salud y Acción Social de la Nación y que por Resolución $\mathrm{N}^{\circ} 530 \mathrm{SC} / 2000$ son de cumplimiento obligatorio, deberán ser adaptados por los titulares de las estaciones radioeléctricas involucradas a fin de cumplir con las condiciones impuestas por dichas resoluciones $[\ldots]^{45}$.

Afirma la Comisión Nacional de Comunicaciones en su publicación denominada "Radiaciones no ionizantes" de febrero de 2007

[...] El marco normativo de nuestro país, sobre los niveles de la Máxima Exposición Poblacional (MEP) a las Radiaciones No Ionizantes, están basados en las últimas recomendaciones de la Organización Mundial de la Salud (OMS). Este organismo es el encargado de orientar y coordinar los estudios científicos, estadísticos y epidemiológicos sobre todo lo que concierne a la protección de la salud y el medio ambiente, generados por los principales centros de investigaciones e instituciones científicas en el mundo, a partir de lo cual realiza determinadas recomendaciones. Con el objeto de asegurar que la exposición humana a los campos electromagnéticos no tenga efectos perjudiciales para la salud, que los equipos generadores de esos campos sean inocuos para la salud, se han adoptado diversas directrices y normas internacionales. Esas normas se elaboran después que grupos de trabajo científicos calificados, que buscan pruebas de la repetición sistemática de efectos perjudiciales para la salud, hayan analizado todos los estudios y las publicaciones científicas con los resultados de institutos de investigación reconocidos. La OMS basa sus recomendaciones

\footnotetext{
${ }^{45}$ Fragmento Resolución 369/2004 de la Comisión Nacional de Telecomunicaciones.
} 
en los estudios de la Comisión Internacional para la Protección contra las Radiaciones No Ionizantes (ICNIRP) [...] A su vez la ICNIRP es una organización científica independiente con el mandato de la OMS para proveer pautas y recomendaciones sobre los peligros para la salud de la exposición a las RNI [...];

[...] En la República Argentina el Ministerio de Salud y Acción Social de la Nación, mediante Resolución 202 del año 1995 establece los valores de Máxima Exposición Poblacional (MEP) para las Radiaciones No Ionizantes, los cuales están por debajo de lo que con posterioridad recomendó la OMS $[\ldots]^{46}$

El parágrafo transcripto nos permite visualizar de qué modo las normativas nacionales se relacionan con las proposiciones de carácter internacional, en razón de lo cual se afirma que nuestras normas internas son reflejas en relación a las recomendaciones provenientes de la OMS que, a su vez, se fundan en los criterios propugnados por parte de la ICNIRP.

\subsection{Una mirada a "gran escala"}

A un nivel más micro, en la Provincia de Santa Fe, se sancionó la Ley de Telefonía Celular Na 12.362/2004, en la cual se verifica la lógica de estandarización dentro de las condiciones de habilitación de antenas estipuladas. Su segundo artículo afirma que

[...] A los efectos de solicitar la habilitación para la instalación, las empresas prestatarias del servicio de telefonía celular deberán presentar ante los Municipios y/o Comunas, lo siguiente:....bUn informe que contenga el cálculo de la densidad de potencia que aproximadamente emitirá la antena a instalar. Dicho informe de cálculo, deberá ser compatible con los estándares establecidos por las normas nacionales vigentes - Resolución N²02/95 del Ministerio de Salud y Acción Social de la Nación y Resolución No 530/2000 de la Secretaría de Comunicaciones de la Nación, o los parámetros que a futuro determinen los organismos anteriormente mencionados, o los que tuvieren competencia en el tema, acorde a los lineamientos establecidos por organismos competentes internacionales $[\ldots]^{47}$

\footnotetext{
${ }^{46}$ Fragmento de la publicación COMISIÓN NACIONAL DE COMUNICACIONES DEPENDIENTE DE LA SECRETARÍA DE COMUNICACIONES DEL MINISTERIO DE PLANIFICACIÓN FEDERAL, INVERSIÓN PÚBLICA Y SERVICIOS. Radiaciones no ionizantes. [S.1.], feb. 2007.

${ }^{47}$ Fragmento de la Ley de Telefonía Celular de la Provincia de Santa Fe Na 12.362/2004.
} 
Por su parte, en el artículo siguiente, se establece la obligatoriedad de presentación de un plan de desarrollo técnico para la prestación del servicio que “[...] contemplará la estrategia a seguir en cada localidad y con proyección de 4 (cuatro) años [...]"48 y deberá indicar entre otras características la densidad máxima de potencia irradiada, la distancia desde donde está tomada dicha densidad de potencia instalada (la vivienda más próxima o la vía pública), la frecuencia de transmisión de cada antena y la ubicación (en metros) de la o las antenas respecto a la antena más próxima, propia o de terceros.

Lo expuesto, se refuerza con las normas de seguridad que prevé la ley, entre las cuales se obliga a las empresas prestatarias a presentar periódicamente por ante el órgano municipal

[...] a) Un padrón actualizado de antenas con sus respectivas potencia de emisión y direcciones; b) Un mapeo de toda la localidad, donde se determine la inmisión en diversos puntos de la urbe y constatar así si existen niveles mayores a los tolerados por Resolución N² 202/95 del Ministerio de Salud y Acción Social de la Nación; c) A través de este mapa se analizará cada caso puntual y se determinará si corresponde el otorgamiento de permisos para el emplazamiento de la antena o el soporte de la misma, en función de los niveles tolerados por Resolución N² 202/95 del Ministerio de Salud y Acción Social de la Nación; d) Informes sobre los valores finales de radiación efectivamente emitidos o a emitir por las antenas ya instaladas o a instalar. Las mediciones se deberán determinar con instrumentos que tengan certificación reconocida de calidad y llevadas a cabo por organismos públicos reconocidos en la materia $[\ldots] .{ }^{49}$

También a nivel aún más micro, podemos visibilizar la estrategia propuesta por el discurso de las agencias globales en la determinación del contenido de las normas, en este caso, municipales.

Es así como nos hallamos con la Ordenanza Na 10.578/2000 que regula la materia de antenas de telefonía móvil en la ciudad de Santa Fe. En ésta también se solicita “[...] h) Informe del cálculo de la densidad de potencia que aproximadamente emitirán las antenas a instalar, realizado por organismo técnico oficial com-

\footnotetext{
${ }^{48}$ Fragmento de la Ley de Telefonía Celular de la Provincia de Santa Fe Na 12.362/2004.

${ }^{49}$ Fragmento del artículo 5 Ley de Telefonía Celular de la Provincia de Santa Fe Na $12.362 / 2004$.
} 
petente $[\ldots]]^{50}$, así como también se requiere una evaluación de impacto ambiental en el art. 10 que establece que

[...] Para la evaluación del impacto ambiental por exposición a radiaciones no ionizantes, radiofrecuencias y microondas, se adoptará como normativa la Resolución № 202/95 del Ministerio de Salud y Acción Social de la Nación a la que adhirió la Provincia de Santa Fe mediante Decreto No 1.532/97. De normarse nuevos y más restrictivos estándares, deberán ajustarse a los mismos. Para la evaluación por perturbaciones a nivel sonoro, será de aplicación la Ordenanza No 9.623/92 [....$^{51}$

Ahora bien, si nos circunscribimos al recorrido efectuado hasta aquí, podríamos pensar que la lógica de estandarización se halla linealmente extendida desde la esfera internacional a la estatal y local. Sin perjuicio de ser esta la mecánica dominante, existen algunos casos en los que se plantean situaciones alternativas.

Dentro mismo de la Provincia de Santa Fe, nos encontramos con varias ciudades que han prohibido la instalación de este tipo de antenas. Por ejemplo, en la localidad de Firmat,

[...] Con el propósito de proteger el medio ambiente y la salud de la población, el Concejo Municipal de esta ciudad sancionó una ordenanza que prohíbe la instalación de antenas de telefonía celular dentro del radio urbano. La normativa es similar a la que aplicaron otras localidades santafesinas donde también se intenta evitar posibles contaminaciones electromagnéticas generadas por las radiaciones que emanan estos aparatos [...]. ${ }^{52}$

Es así como dentro de este escenario de "expansión lineal”, se advierten algunas alternativas de "resistencia local"53. En casos como el último que reseñamos, se ha decidido no ingresar en esta lógica de aceptación del riesgo que pareciera sustentarse en una suerte normalización del mismo a partir del cumplimiento de algunos parámetros de emisión/exposición definidos por el expertise.

Ahora bien, dado el escenario de controversia científica que se presenta en este supuesto, la "desconfianza en la ciencia” podría plantear situaciones de "reconfianza" en

\footnotetext{
${ }^{50}$ Fragmento del art. 8 de la Ordenanza Na 10578/2000 de la ciudad de Santa Fe.

${ }^{51}$ Fragmento del art. 10 de la Ordenanza Na 10578/2000 de la ciudad de Santa Fe.

${ }^{52}$ Fragmento del artículo periodístico "Prohíben la instalación de antenas de telefonía celular en Firmat" publicado en el Diario La Capital en fecha 01.06.06.-

${ }^{53}$ SANTOS, Boaventura de Sousa. A globalizacao e as ciencias sociais. Sao Paulo: Cortéz , 2002.
} 
el sentido común ${ }^{54}$ Lo expuesto, se visibiliza en frases de algunos actores implicados en la temática que afirman “[...] No estoy diciendo que antena sea igual a cáncer. Pero si la presunción de que puede hacer daño existe, entonces hay que sacarla [... $]^{{ }_{55}}$

En consonancia con ello, se torna factible pensar en una "recreación participativa" 56 , que permita proponer normas cuya racionalidad no derive exclusivamente del discurso de las ciencias, es decir, que no se presenten sólo a la manera de juridizaciones del discurso experto. Esta "recreación participativa”, en el caso que nos ocupa, se presenta tanto a nivel institucional como a nivel no institucional. En el supuesto de Firmat, verbigracia, estamos ante un ejemplo de participación institucionalizada, que queda plasmado en la ordenanza que prohíbe la instalación de antenas en la localidad.

Por su parte, se yuxtaponen, también, mecanismos no institucionalizados de participación como, por ejemplo, marchas en diversas localidades bajo la consigna "no a las antenas" ${ }^{37}$.

De este modo, en la "constelación jurídica” a partir de la cual trabajamos, podemos observar que "la pequeña escala del mapa" expande y reproduce su lógica de estandarización como dispositivo precautorio tanto en la "escala media" como en la "gran escala" del mapa analizado. Pero, paralelamente, se visualizan algunos casos puntuales que se presentan como alternativas para asignar tratamiento a una cuestión científicamente controvertida, lo que puede dar lugar a una reinvención de disposiciones y

${ }^{54}$ SANTOS, Boaventura de Souza. Crítica de la razón indolente: contra el desperdicio de la experiencia. Bilbao: Desclée de Brouwer, 2003.

${ }^{55}$ Fragmento del artículo periodístico "A sacar las ondas del colegio" publicado en el diario Página 12 en fecha 26 de octubre de 2006 a propósito de la instalación de una antena de telefonía celular en el techo de la escuela "San José" en la localidad de Villa Crespo, Buenos Aires.

${ }^{56}$ Referimos a "recreación participativa" dentro de la lógica del andamiaje conceptual de Boaventura de Sousa Santos, especialmente en cuanto a su planteo acerca de reinventar la democracia.

${ }^{57}$ Se desarrollan manifestaciones de este tipo en diferentes barrios de la Ciudad Autónoma de Buenos Aires. Por su parte un caso interesante se desarrolló en Pueblo Belgrano (Provincia de Entre Ríos), manifestación a la cual se sumaron miembros de la asamblea ambiental de Gualeguachú, la que surgió a partir de la instalación de dos pasteras frente a la mencionada localidad a la vera del río Uruguay sobre la costa de la República Oriental del Uruguay. Esto ha generado un caso de contaminación transfronteriza, que da paso a una resistencia local no institucionalizada por parte de vecinos de dicha ciudad, que se edifica en concomitancia con el proceso de construcción de las pasteras iniciado hacia el año 1998. 
normas tendientes a la gestión de este riesgo, tal vez más cercanas a la participación por parte de los actores sociales implicados.

\section{Respecto de la identificación del discurso de las agencias globales en la jurisprudencia local}

En esta última parte, nos proponemos rastrear, de modo exploratorio cómo, dentro del marco de algunos casos jurisprudenciales argentinos en materia de campos electromagnéticos, se identifican, también, referencias al discurso elaborado por parte de las agencias de gobierno global.

Esta es otra de las vías a partir de las que se puede visibilizar la penetración de los discursos provenientes de las agencias globales al ámbito local.

Específicamente, en relación a la instalación de antenas de telefonía celular ${ }^{58}$, nos hallamos con dos sentencias en las que se hace referencia explícita al discurso internacional como un argumento relevante al momento de resolver el supuesto de hecho bajo análisis.

\footnotetext{
${ }^{58}$ Existen otras sentencias sobre campos electromagnéticos en que se hace referencia al discurso de la OMS - ICNIRP, pero en relación a plantas transformadoras de energía eléctrica, que configuran otro de los agentes emisores de radiaciones no ionizantes. Es así como referimos a la sentencia de la Causa 3801/02 de la Cámara Federal de Apelaciones de La Plata - Sala II 08.07.2003. "Asociación Coordinadora de Usuarios, Consumidores y Contribuyentes c/ ENRE - EDESUR s/ cese de obra de cableado y traslado de Subestación Transformadora" en la que se afirma "[...] En tal sentido, señala que la empresa mantiene actualmente la potencia originaria que la subestación Sobral sustentó desde que fuera puesta en marcha por SEGBA en el año 1982. Agrega que, además de haber sido aprobadas las instalaciones de la planta por las autoridades competentes tanto nacionales como municipales, las mediciones realizadas entre los años 1998 y 2002 por EDESUR y el ENRE, con la cooperación de la Universidad Nacional de La Plata, cumplen con la normativa nacional específica (Resol. 77/98 de la S.E.) y con los valores de guía internacionales fijados por la ICNIRP (Comisión Internacional de Protección contra Radiaciones No Ionizantes) reconocidos por la Organización Mundial de la Salud. De manera tal que, la actividad desplegada por la empresa demandada se ajusta cabalmente a la normativa específica aplicable (leyes 14.772 y 15.336 -art. 37-;; decreto 714/92 y Resolución S.E. 77/98 -4.5.1-) [...]”. Por su parte, en la sentencia de la Cámara Federal de La Plata - Sala I - 30.08.2007 "Edesur S.A. c/ Municipalidad de Berazategui" se sostiene que "[...] La Hoja Informativa n. 263 del Proyecto Internacional CEM, publicada en el mes de octubre del 2001, da cuenta de una reciente investigación llevada a cabo por la Agencia Internacional para la Investigación del Cáncer (IARC) -una agencia de la OMS especializada en la investigación de esa enfermedad- que concluyó el primer paso sobre el proceso de evaluación del riesgo a la salud de los campos electromagnéticos de frecuencia extremadamente baja, clasificando dichos campos con respecto a la fuerza de la evidencia como que podrían causar cáncer en humanos [...].”
} 
En primer lugar, la sentencia del Tribunal Superior de Córdoba, de fecha 11.03.2003, caratulado "Castellani, Carlos y E. y otros s/ amparo", que se inicia a partir de una acción de amparo presentada por un grupo de vecinos de la localidad de Oncativo a los fines de solicitar se impida la instalación de cuatro antenas de telefonía móvil.

En ésta el Tribunal, en el voto de la mayoría, remite al Dictamen de la Comisión de Evaluación sobre la Contaminación Visual, Sonora y Electromagnética de las Antenas de Comunicación que se sustenta, a su vez, en el discurso de la OMS, afirmando que

[...] El balance de las evidencias recogidas sugiere que la exposición a la radiación de radiofrecuencia en las frecuencias utilizadas por los emisores de la telefonía móvil, dentro de los límites recomendados por la NRPB y ICNIRP no causan efectos adversos a la salud de la población en general [...]. Los vacíos de conocimiento en varias áreas son suficientes para que en este momento no sea posible asegurar que la exposición a radiación $\mathrm{RF}$, aún para niveles inferiores a los más exigentes recomendados internacionalmente, carece completamente de efectos potencialmente adversos para la salud $[\ldots]^{59}$.

El Tribunal decide no hacer lugar al amparo debido a que no existen pruebas suficientes que permitan sostener el posible acaecimiento de daños para la salud de las personas involucradas.

Similar referencia a lo sostenido desde las agencias globales, se repite en la sentencia del Juzgado de Primera Instancia en lo Civil $\mathrm{N}^{\circ} 105$ de Buenos Aires, de fecha 09.02.2007, caratulado "Espíndola María c/ Movicom Bellsouth s/ Daños Perjuicios” en la que se argumenta

[...] Que con el fin de dar cumplimiento con las necesidades expuestas, se dictó la RESOLUCION 269 del 18 de marzo de 2002, modificada por la RESOLUCION 117 de fecha 24 de enero de 2003, ambas de la COMISION NACIONAL DE COMUNICACIONES. Que los procedimientos se basan en la normativa internacional en la materia, tal como la COMISION INTERNACIONAL DE PROTECCION CONTRA RADIACIONES NO IONIZANTES (ICNIRP), la Unión Internacional de Telecomunicaciones (Recomendación UIT- K-61), el Comité, Electrotécnico

\footnotetext{
${ }^{59}$ ARGENTINA.Tribunal Superior de Córdoba. Sentencia 664 Castellani, Carlos y E. y otros, responsabilidad civil y seguros. Buenos Aires, 11 de março de 2003. Buenos Aires, v. 5, n. 6, nov./dic. 2003
} 
Internacional (Norma Internacional 61.566/1997) [...]. Que asimismo, se ha tenido en cuenta la opinión de organismos nacionales con competencia en la materia. Que, así también, se ha determinado, la importancia de unificar criterios en el orden nacional, con especial énfasis en jurisdicciones municipales, a efectos de concretar un procedimiento uniforme para el control de las radiaciones no ionizantes $[\ldots] .^{60}$

A diferencia del supuesto anterior, aquí se ordena no sólo la desconexión de la antena sino también la indemnización por el posible daño a la salud de la accionante ${ }^{61}$, lo cual remite, aunque tangencialmente a la aplicabilidad al caso de la lógica de precaución.

A partir del análisis de los reseñados casos jurisprudenciales, podemos dar cuenta de cómo el discurso experto funciona a los efectos de sustentar decisorios en ámbitos en que el supuesto de hecho es circundado por un contexto de controversia científica. En ambos, aparece como relevante la estrategia de estandarización basada en la propuesta de parte de la agencia de gobierno global, específicamente, la OMS, cuyos parámetros, como ya hemos señalado,

${ }^{60}$ ARGENTINA. Cámara Federal de Apelaciones de La Plata. Juzgado de Primera Instancia. Sentencia $n .^{\circ} 105$ de 9 de fevereiro de 2007 en causa 17008/03 de Buenos Aires. Espíndola María c/ Movicom Bellsouth s/ Daños Perjuicios. Disponível em: <www.diariojudicial. com.ar > Acesso em: Junio 2007.

${ }^{61}$ Sostiene el juez en este caso: “[...] DAÑOS POTENCIALES A LA SALUD: La actora es una persona de avanzada edad, no vidente, que se ha visto expuesta a esta situación no solo de intranquilidad permanente por los ruidos ocasionados por los equipos, sino también, por los estruendos y fuertes vibraciones, producidos en los días de tormenta por contar la antena con un pararrayos. Por otra parte teniendo en consideración, la pericia efectuada por el Dr. Raul Grigera, Doctor en Física Biológica e Investigador Superior del CONICET quien opina que los "daños en la salud producidos por radiaciones no ionizantes se agravan en caso que se traten de ancianos, niños y personas que posean una sensibilidad especial al efecto de las radiaciones no ionizantes, por lo que una exposición continua, aun por debajo de los límites mencionados, son potencialmente peligrosos". Por lo que fijo prudencialmente el monto de la indemnización correspondiente al daño a la salud, la suma de PESOS CINCO MIL (\$5000), con mas sus intereses que se computar n desde la fecha de instalación de la antena autosoportada (febrero de 1998) hasta su efectivo pago, sobre la tasa de interés pasiva que suministra el Banco Central de la República Argentina [...]" (Fragmento de la sentencia "Espíndola María c/ Movicom Bellsouth s/ Daños Perjuicios"). Es interesante destacar que esta afirmación, que refiere a la resarcibilidad de un daño potencial, no se condice con la estructura tradicional a partir de la cual se edifica la tesis de la resarcibilidad del daño, cuya característica fundamental es la certeza de su acaecimiento. 
se sustentan en las conclusiones elaboradas por el grupo de expertos nucleado bajo la ICNIRP.

De este modo, frente a un caso de controversia científica vemos como el discurso experto, específicamente el proveniente de recomendaciones de alcance global, ha de sustentar decisiones tanto desde una perspectiva tolerante, como sucede en "Castellani”, como desde una racionalidad precautoria, como se visualiza en "Espíndola".

Ello puede correlacionarse con que el estado de controversia científica dificulta la realización de un razonamiento lineal de tipo reflejo en relación al conocimiento experto. Por el contrario, existen diferentes miradas que fundan un decisorio en uno u otro sentido.

Se distingue una postura precautoria, generadora de un "espacio de cautela"; y una posición tolerante que trata de seguir adelante con las actividades posiblemente riesgosas hasta tanto exista certeza sobre su dañosidad, dado que en base a esta lógica no se actúa conforme a conjeturas sino a certitudes.

Ahora bien, el problema que se presenta es similar en los dos casos. Se trata de determinar si el hecho de la generación de ondas electromagnéticas puede configurar un daño a la salud o no. Las decisiones adoptadas son disímiles. La causa de esta discordancia se encuentra signada por la dificultad para gestionar y dar tratamiento a la controversia, en un sistema jurídico que ha sido edificado a partir de la certidumbre, opacando y ocultando todo aquello que se alejara del marco de lo cognoscible.

En un marco controvertido, tanto la opción precautoria como la tolerante pueden hallar sustento en el discurso experto referenciado, al cual se suman informes periciales, sin otorgar mayor relevancia a las manifestaciones de otros actores sociales que no forman parte de la comunidad científica.

Ello se contrapone con la idea de construcción de espacios para una toma de decisiones más democrática, que reflexione sobre las percepciones y posiciones esgrimidas por quienes son pasibles de ser afectados por un hecho cuyas consecuencias dañosas aún no han sido determinadas. En armonía con esta idea, se sostiene que el proceder experto debe ser conocido e integrado en un debate democrático que constituya un medio privilegiado para acercar a la ciencia y al ciudadano. ${ }^{62}$

${ }_{62}$ PIVOT, Catherine. Vers un nouveau mode d'action collective face aux risques. In : 
De este modo, se destaca que es posible asumir formas participativas para dar tratamiento a la incertidumbre, logrando una apertura del marco de la toma de decisiones en relación a quienes posiblemente se encuentren afectados por un riesgo en su salud o en el medio ambiente en el que habitan. Dentro de esta tendencia se hace referencia a los denominados "forum híbridos". Éstos se hallan caracterizados, en líneas generales, por la diversidad de actores que lo integran, dentro de los cuales se destaca un polo tecnocientífico, uno representativo del derecho y otro del mundo sociopolítico y económico ${ }^{63}$ Una idea similar presenta la organización sistemática del expertise en "dos círculos", planteada por P. Kourilsky y G. Viney en el Reporte que sobre el principio de precaución presentaron al Primer Ministro francés en 1999. El primer círculo se integra por expertos que tienen a su cargo el análisis científico y técnico para graduar la hipótesis de riesgo; el segundo se compone de representantes del primero, economistas, actores sociales y representantes del público, que se encargan de debatir las conclusiones a las que arribó el grupo de expertos del primero.

Para finalizar, se destaca la trascendencia de otro elemento que es la construcción de un ámbito de revisión de las decisiones en estos casos en los cuales no hay certeza sobre la dañosidad de un hecho. Se entiende por tal "un reexámen periódico en vista a la adquisición de nuevos datos científicos, lo que significa, al mismo tiempo, que a ello deben seguir nuevas investigaciones y eventuales revisiones que puedan derivar de la evolución del propio conocimiento científico" ${ }^{64}$

La construcción de un espacio de revisión genera la posibilidad de elegir, reelegir o modificar el paradigma en funcionamiento respecto de un caso determinado, a partir de nuevos datos científicos disponibles; con la aptitud para generar un diálogo entre el ámbito experto y el no experto, en el marco de un debate construido a partir de valores plurales y democráticos.

RYCHEN, F. ; PIVOT, C. Gérer les risques Collectifs. Paris: Horizon Groupe, 2002.

${ }^{63}$ CALLON, Michel ; RIP, Arie. Humains, non - humains: morale d'une coexistence en La terre outragée: les experts sont formels. Paris: Autrement, 1996.

${ }^{64}$ NOIVILLE, Christine. Science, décision, action: trois remarques à propos du principe de précaution. Petites affiches, Paris, v. 2, n. 218, Nov., 2004. 


\title{
8 Algunas reflexiones finales
}

Luego de este breve recorrido respecto de la "constelación jurídica" que se edifica a partir de la implementación de estándares, podemos visualizar que el caso bajo estudio deja traslucir como los discursos de las agencias de gobierno global, es decir los dispositivos jurídicos que se corresponden con la "pequeña escala" del derecho; se reproducen luego en la "escala media" y en la "gran escala".

Es así como, desde el derecho "soft" global, se realizan recomendaciones que luego han de condicionar las normativas nacionales e incluso, los trámites de habilitación locales. Ahora bien, también aparecen algunas alternativas a esta lógica aparentemente lineal. Tal y como hemos referenciado, en algunas situaciones este "orden" de la constelación se desarticula y se rearticula a partir de otras variantes, las cuales se relacionan, fundamentalmente, con algunos signos incipientes de mayor participación de parte de los actores sociales implicados, redescubriendo que el sentido común no ha perdido su sentido, sino que necesita ser reinventado ${ }^{65}$ para ser incorporado en los diseños de políticas de gestión de riesgos.

De este modo, se trata de "rearticular la constelación", dejando su lugar al discurso experto, pero permitiendo que a su lado se incorporen otros tipos de discursos que problematicen la idea de estandarización como una alternativa posible, dando apertura a un marco de posibilidades más amplio y, por qué no, más creativo.

\section{Standardization as a globalised precautionary technology: a case study on non-ionizing radiation emission}

\begin{abstract}
The standardization strategy is a juridical tool able to start, expand and develop precautious rationality, which is fundamentally linked to environmental government of risks and related to human health. In this work, we propose the establishment of limits for non ionizing radiation emission, a globalization process for the social-juridical field. We started from the idea proposed by the sociologist Boaventura de Sousa Santos about the coexistence of different normative
\end{abstract}

${ }^{65}$ SANTOS, Boaventura de Souza. Crítica de la razón indolente: contra el desperdicio de la experiencia. Bilbao: Desclée de Brouwer. 2003 
production levels (international/national/local) with the aim of establishing the relationships and articulation between these levels in the case proposed. In order to do this, we carried out a survey of all the existing regulations regarding the establishment of maximum emission and exhibition levels enlisted in each of these normative levels and we also analyzed how each of them operate the jurisprudence related to the chosen thematic, specifically, in respect to the installation of cellular telephone antennas. This analysis is formulated as an attempt to give visibility to the articulation and assemblies necessary for the establishment of nonionizing radioactivity emission limits, in order to contribute to the debate about the process of globalization in the field of law.

Keywords: Globalization. Law. Environmental Law.

\section{Referências}

A SACAR las ondas del colegio. Diario, Buenos Aires, p. 12, 26 out. 2006.

ARGENTINA. Cámara Federal de Apelaciones de La Plata. Sentencia de la Causa no 3.801/02 de la Cámara Federal de Apelaciones de La Plata de 8 de julho de 2003. Asociación Coordinadora de Usuarios, Consumidores y Contribuyentes c/ ENRE - EDESUR s/ cese de obra de cableado y traslado de Subestación Transformadora. Disponível em: <www.lexisnexis.com.ar >. Acesso em: Jun. 2006

. Juzgado de Primera Instancia. Sentencia $n .{ }^{\circ} 105$ de 9 de fevereiro de 2007 en causa 17008/03 de Buenos Aires. Espíndola María c/ Movicom Bellsouth s/ Daños Perjuicios. Disponível em: <www.diariojudicial.com.ar > Acesso em: Junio 2007.

ARGENTINA. Ley de Política Ambiental n o 25675 2002. Disponível em: <www. infoleg.com>.

Ley de Telefonía Celular $n^{\circ}$ 12.362. Santa Fe, 2004. Disponível em: <www. infoleg.com $>$.

Ordenanza $n^{\circ}$ 10578. Santa Fe, 2000. Disponível em: <www.santafeciudad. gov.ar>.

Sentencia de la Cámara Federal de La Plata de 30 de agosto de 2007. Edesur S.A. c/ Municipalidad de Berazategui. Disponível em: <www.lexisnexisonline>. Acesso em: Nov. 2007. 
. Tribunal Superior de Córdoba. Sentencia 664. Castellani, Carlos y E. y otros, responsabilidad civil y seguros, Buenos Aires, 11 de março de 2003. Buenos Aires, v. 5, n. 6, nov./dic. 2003.

BECK, Ulrich. La sociedad del riesgo global: siglo XXI. [S.l: s.n.], 2002.

. La sociedad del riesgo mundial. [S.1.]: Paidós, 2007.

. La sociedad del riesgo: hacia una nueva modernidad. [S.1.]: Paidos, 1998.

CALLON, Michel; RIP, Arie. Humains, non-humains: morale d'une coexistence en La terre outragée. Les experts sont formels. Paris: Autrement, 1996.

CARR, S; IBARRA, A. Las construcciones del riesgo en Gobernar los riesgos: ciencia y valores en la sociedad del riesgo. Madrid: Biblioteca Nueva OEA, 2004.

COMISIÓN NACIONAL DE COMUNICACIONES DEPENDIENTE DE LA SECRETARÍA DE COMUNICACIONES DEL MINISTERIO DE PLANIFICACIÓN FEDERAL, INVERSIÓN PÚBLICA Y SERVICIOS. Radiaciones no ionizantes. [S.l.], feb. 2007.

DE SADELEER, Nicolás. Environnemental principles: from political slogans to legal rules. New York: Oxfor University Press, 2002.

DOUGLAS, Mary. La aceptabilidad del riesgo según las ciencias sociales. Buenos Aires: Paidos, 1996.

DRNAS, Zlata de Clement. El principio de precaución ambiental: la práctica argentina. Córdoba: Lerner SRL, 2007.

ESTEVE PARDO, José. Técnica, riesgo y derecho: tratamiento del riesgo tecnológico en el derecho ambiental. Barcelona: Ariel, 1999.

EWALD, François. L'État de précaution. Rapport public du Conseil de Etat. Jurisprudence et avis de 2004. Responsabilité et socialisation du risque. La Documentation Française: Etudes et Documents, Paris, n. 56, 2005.

. L'Etat Providence. Paris: Grasset, 1986.

EWALD, François. La société du risque en une nouvelle modernité? Traitements de surface et exploration des profondeurs. Descartes \& Cie: Cahier LaSer, Paris, n. 7, p. 105-130, 2001. 
Le principe de précaution: que sais je? Paris: PUF, 2001.

. Le retour du malin génie: esquisse d'une philosophie de la précaution. In: GODARD, Olivier (Ed.). Le principe de précaution dans la conduite des affaires humains. Paris: Maison des Sciences de l'homme 1997. p. 99-126.

Le risque dans la société contemporaine. Université de tous les savoirs sous: qu'est - ce les technologies? Paris, v. 5, 2000. $412,1996$.

. Philosophie de la précaution. L’Année sociologique, Paris, n. 46, p. 384-

. Responsabilité, solidarité, sécurité. Risques. Assurance, droit, responsabilité, Paris, n. 10, Avr./Jui. 1992.

GIDDENS, Anthony et al. Las consecuencias perversas de la modernidad. Barcelona: Anthropos, 1996.

GODARD, Olivier. L'ambivalence de la précaution et la transformation des rapports entre sciences et décision. In: GODARD, Olivier (Ed.). Le principe de précaution dans la conduite des affaires humains. Paris: Maison des Sciences de l'homm, 1997. p. 37-83.

HERMITTE, Marie-Angèle. Fundación jurídica de una sociedad de las ciencias y de la técnica a través de las crisis y los riesgos. In: VARELLA, M. Derecho, sociedad y riesgos: la sociedad contemporánea vista a partir de la idea de riesgo. Red Latinoamericana y Europea sobre Gobierno de los Riesgos. Brasilia: Uniceub, 2006.

. La sang et le droit. Paris: Seuil, 1998

- Le principe de précaution à la lumière du drame de la transfusion sanguine en France. In: GODARD, Olivier (Ed.). Le principe de précaution dans la conduite des affaires humains. Paris: Maison des Sciences de l'homme, 1997. p. 179-198.

JONAS, Hans. Le principe de responsabilité. Paris: Du Cerf, 1990.

KEMELMAJER DE CARLUCCI, Aida. El principio de precaución en un documento de la UNESCO. Academia Nacional de Derecho y Ciencias Sociales de Buenos Aires: Anticipo de Anales, Buenos Aires, v. 50, n. 43, 2006. 
LATOUR, Bruno. Nous niavons jamais été moderne: essai d'anthropologie symétrique. Paris: La Decouverte, 1997.

NOIVILLE, Christine. Science, décision, action: trois remarques à propos du principe de précaution. Petites affiches, Paris, v. 2, n. 218, Nov. 2004.

ORGANIZACIÓN MUNDIAL DE LA SALUD. [Home page]. Disponível em: <www.who.int> Acesso em: 19 jul. 2008.

. Legislación Modelo para la Protección contra Campos Electromagnéticos = Model Legislation for Electromagnetic Fields Protection. Trad. Víctor Cruz Ornetta et al. Lima: INICTEL; UNI, 2007.

- Marco para el desarrollo de estándares de CEM basados en la salud: framework for developing health - based EMF standards. Trad. Víctor Cruz Ornetta. Lima: INICTEL; UNI, 2007.

PIVOT, Catherine. Vers un nouveau mode d'action collective face aux risques. In : RYCHEN, F. ; PIVOT, C. Gérer les risques Collectifs. Paris. Horizon Groupe, 2002.

PROHÍBEN la instalación de antenas de telefonía celular en Firmat. Diario La Capital, Buenos Aires, 01 jun. 2006.

RÈMOND-GOUILLOUD, Martine. El derecho a destruir: ensayo sobre el derecho del medio ambiente. Buenos Aires: Lozada, 1994.

SANTOS, Boaventura de Sousa. A globalizacao e as ciencias sociais. Sao Paulo: Cortéz, 2002.

. Crítica de la razón indolente: contra el desperdicio de la experiencia. Bilbao: Desclée de Brouwer, 2003.

- La globalización del derecho: los nuevos caminos de la regulación y la emancipación. Bogotá: Facultad de Derecho Cs Políticas y Sociales, Universidad Nacional de Colombia, 2002.

SOZZO, Gonzalo (Coord.). El gobierno de los riesgos. Santa Fé: Universidad Nacional del Litoral. 2007.

SOZZO, Gonzalo. La prevención del daño (ensayo desde la mirada de las vías periféricas). Revista de derecho privado y comunitario, Santa Fe, n. 2 p. 391-421, Sep. 2008. 
Repensar la regla de la asunción del riesgo: de causal de justificación a dispositivo tecnológico de gobierno de los riesgos. Revista de Derecho de Daños, Santa Fe, n. 2, p. 287-343, Sep. 2007.

- Riesgos del desarrollo y sistema del derecho de daños (hacia un derecho de daños pluralista). In: VARELLA, Marcelo Dias (Coord.). Direito, sociedade e riscos: sociedade contemporânea vista a partir da idéia de risco Brasilia: Red Latino-Americana e Européia sobre Governo dos Riscos, 2006.

ORGANIZACIÓN MUNDIAL DE LA SALUD. Legislación modelo para la protección contra campos electromagnéticos: model legislation for electromagnetic fields protection". Trad. Víctor Cruz Ornetta et al. Lima: INICTEL-UNI, 2007. Data de acesso: 19 jul. 2008. 